\title{
ESTUDO COMPARATIVO ENTRE AS TÉCNICAS MANUAL EAUTOMÁTICA DE DEMARCAÇÃO DE BORDA PARA AVALIAÇÃO DE ÁREA DE ÚlCERAS POR PRESSÃO
}

Karolina Bastos da Cruz Salmonaํ, Levy Aniceto Santana ${ }^{2}$, Rinaldo de Souza Neves ${ }^{3}$, Renato da Veiga Guadagnin ${ }^{4}$

Objetivo: comparar a acurácia entre a técnica manual e automática de demarcação da borda da ferida para cálculo de área pelo software ImageJ para avaliação de úlceras por pressão (UP). Metodologia: trata-se de um estudo transversal controlado com análise computacional de imagens realizada em 35 fotografias digitais de úlceras por pressão (UP) localizadas nas regiões sacral, trocanteriana e isquiática. O software utilizado para cálculo de propriedades (ImageJ) é um recurso não invasivo, reprodutivel, objetivo e de uso viável na prática clínica para delimitação da área de feridas. Utilizou-se o software SPSS (Statistical Package for the Social Sciences) para a análise descritiva dos dados, concordância entre as medidas pelo procedimento de Bland e Atman. Resultados: observou-se pouca concordância entre as técnicas. A técnica automática denominada Brightness/Contrast demonstrou medidas mais aproximadas à técnica manual para avaliação de área de UP. Conclusão: ambas as técnicas computacionais, manual e automática do software ImageJ, para cálculo de área de UP, demonstraram boa correlação positiva, mas também pouca concordância entre as técnicas, quando comparadas.

Descritores: Cicatrização de Feridas; Úlceras por Pressão; Avaliação

\section{COMPARATIVE STUDY BETWEEN MANUAL AND AUTOMATIC TECHNIQUES TO PINPOINT WOUND EDGES DURING PRESSURE ULCERS PERIMETER EVALUATION}

Objective: to compare the accuracy between manual and automatic perimeter estimation technique to pinpoint wound edges using the software ImageJ to ascertain pressure ulcers (PU). Methodology: through a controlled transversal investigation using 35 pressure ulcers (PU) digital photographs taken in the sacral, trochanter and ischium areas. The software used to determine its properties (ImageJ) is a noninvasive, objective and reproducible practical resource to use in clinical practice to delimit the wound area. The SPSS (Statistical Package for the Social Sciences) software made possible a descriptive analysis of data and concordance between the measurements using Bland and Altman procedure. Results: a slight agreement between the techniques has been discovered. The automated technique known as Brightness/Contrast displayed results closer to the manual technique to estimate PU area. Conclusion: both computational techniques, manual and automatic, of the ImageJ software to estimate PU area showed sound positive correlation, but also limited correlation between the techniques, when cross-checked.

Descriptors: Wound healing; Pressure ulcers; Measurement.

\section{INVESTIGACIÓN COMPARATIVA ENTRE LASTÉCNICAS MANUALYAUTOMÁTICA DE DEMARCACIÓN DE BORDE PARAEVALUACIÓN DELÁREA DE ÚLCERAS POR DECÚBITO}

Objetivo: comparar la precisión entre la técnica manual y automatizada de demarcación de borde de la lesión para calcular el área por el software ImageJ para evaluación de úlceras por decúbito (UD). Metodología: tratase de una investigación transversal manejada con el análisis computacional de imágenes de 35 fotografias digitales de úlceras por decúbito (UD) localizadas en las regiones sacra, regiones trocanteras e isquiática. El software utilizado para el cálculo de propiedades (ImageJ) es un recurso no invasivo, reproducible, objetivo e de uso manejable en la práctica clínica para la demarcación del área de las lesiones. Se aplicó el software SPSS (Statistical Package for the Social Sciences) para el análisis descriptivo de los datos, conformidad entre las mediciones por intermedio del método Bland y Atman. Resultados: se constató conformidad limitada entre las técnicas. La técnica automática denominada Brightness/Contrast demostró mediciones más próximas a la técnica manual para evaluación del área UD. Conclusión: las dos técnicas computacionales, manual y automatizada del software ImageJ, para el cálculo del área UD, han revelado gran correlación positiva, pero también poca correlación entre las técnicas, cuando comparadas.

Descriptores: Curación de Heridas; Úlceras por Decúbito; Evaluación.

${ }^{1}$ Fisioterapeuta. Graduada pela Universidade Católica de Brasilia.

${ }^{2}$ Fisioterapeuta. Doutor em Ciências da Saúde. Docente da Escola Superior de Ciências (FEPES).

${ }^{3}$ Enfermeiro. Doutor em Ciências da Saúde. Docente da FEPES. E-mail: rinaldodesouza@gmail.com

${ }^{4}$ Engenheiro Mecânico. Doutor em Ciências da Administração. Docente do Programa de Mestrado em Gestão do Conhecimento e da Tecnologia da Informação da Universidade Católica de Brasilia. 


\section{INTRODUÇÃO}

As úlceras por pressão (UP) são definidas como qualquer alteração relacionada à integridade tegumentar, resultantes da compressão não aliviada de tecidos moles entre uma proeminência óssea e uma superfície rígida. Compreendem uma das principais complicações entre os pacientes críticos, sendo o tratamento, em geral, prolongado e oneroso ${ }^{(1,2)}$.

Dentre os fatores de risco intrínsecos e extrínsecos predisponentes às feridas, destacam-se: idade avançada, imobilidade, alterações de sensibilidade e consciência, bem como a sua associação à umidade, cisalhamento e fricção(3). Em uma Unidade de Terapia Intensiva Adulto de um serviço de Saúde Pública do Distrito Federal (DF), no ano de 2010, foram identificadas incidência de $37 \%$ e prevalência de $57,9 \%$ de UP(4).

Considerada como um indicador de qualidade na saúde, ora relacionado às intervenções implementadas em situações de hospitalização e/ou institucionalização, ora relacionado à prevenção, a ferida constitui um transtorno significativo à medida que promove redução relevante da qualidade de vida do paciente. Estima-se que $0,4 \%$ a $38 \%$ de indivíduos hospitalizados desenvolvem UP, sendo esse um problema de saúde persistente $e^{(5,6)}$.

A monitorização e avaliação precisa da extensão da ferida permite ao profissional definir prognóstico, desenvolver intervenções terapêuticas efetivas baseadas em tomadas de decisão clínica, com fundamento em evidências e, além disso, melhorar os cuidados assistenciais ao paciente ${ }^{(7)}$.

Existem diversas técnicas para aferição de área de feridas, dentre elas, a planimetria manual, método quantitativo simples, que utiliza um material transparente, como uma folha de acetato. É um procedimento rápido e de baixo custo, semelhante a uma avaliação usando uma régua. Já a planimetria digital, com análise do traçado manual da ferida em escâner portátil, permite maior precisão da área de interesse automaticamente ${ }^{(7)}$.

A evolução tecnológica e o uso de fotografias digitais permitem o registro do objeto e análise computadorizada por software especializado de forma precisa, não invasiva e com riscos reduzidos de contaminação, sendo amplamente utilizado para acompanhamento da evolução clínica das feridas ${ }^{(2,7)}$.

Encontra-se disponível comercialmente uma variedade de softwares editores de imagem digital para o acompanhamento de área topográfica e também de feridas, os quais podem ser úteis em diversas áreas do conhecimento, como engenharia civil, odontologia e oftalmologia. Dentre esses softwares, o AutoCAD ${ }^{\circledR}$ (Autodesk Inc.) tem sido utilizado em estudos com animais e seres humanos para a avaliação de área de feridas ou UP( ${ }^{(8)}$, assim como o MOTIC ${ }^{\circledR}$ (Instruments Inc. Canadá), que permite aplicação semelhante. Também são descritos na literatura $^{(9-11)}$ os programas VeVMD (Vista Medical, Winnipeg, Manitoba, Canada), desenvolvidos para mensurar e organizar dados obtidos a partir das imagens, e WHAT (Wound Healing Analysing Tool), o qual possibilita a avaliação e submissão de dados e relatórios aos centros de tratamento, via acesso à Internet. No entanto, o custo elevado dos softwares comerciais, bem como a quantidade insuficiente de profissionais com conhecimento para seu manuseio, tornam inviável o emprego maciço do método às práticas clínicas rotineiras.

Dentre os recursos computacionais para a mensuração da área de objetos de interesse, o software ImageJ é considerado como um programa que dispõe de ferramentas úteis para o acompanhamento da evolução cicatricial. É um software gratuito e de livre acesso, que possibilita processar imagens com rotinas existentes (plugins) e a construção de rotinas pelo próprio usuário em linguagem Java ${ }^{\circledR}$. A utilização do ImageJ foi proposta em estudo histopatológico esofágico, sendo considerado reprodutível, objetivo e facilmente aplicável(12).

O ImageJ permite a medição tanto manual, a partir da delimitação da área de interesse com auxílio do mouse, como automática. Efetua a binarização da imagem, transformando-a em tons branco e preto, destacando o objeto de interesse ${ }^{(9,12)}$. A técnica manual é a mais utilizada, cujos resultados demonstram eficácia e boa confiabilidade, apesar de haver contradições no que se refere à habilidade do examinador. Já a técnica automática é pouco encontrada na literatura(7), observadas as dificuldades em caráter prático para a aferição de área das feridas.

Nesse contexto, o objetivo desse estudo é comparar o uso das técnicas manual e automática de demarcação de borda para avaliação de área de UP pelo software ImageJ.

\section{METODOLOGIA}

Trata-se de estudo transversal controlado com análise computacional de imagens, em 35 fotografias digitais de portadores de UP, captadas em uma unidade de internação de um hospital público da Secretaria de Estado de Saúde do Distrito Federal (SES/DF), no período de fevereiro a julho de 2013. Foram incluídos 18 pacientes portadores de UP em região sacral, trocantérica e isquiática e excluídos da amostra os que apresentavam essas UP com fístula ou túnel, pois essa alteração dificultaria a mensuração das bordas e de suas áreas.

O protocolo experimental foi aprovado pelo Comitê de Ética em Pesquisa (CEP) da Fundação de Ensino e Pesquisa em Ciências da Saúde (FEPECS) da SES/DF, número 192/641. Utilizou-se um formulário para obter dados de identificação 
do paciente, assim como da idade, tempo médio da lesão, sexo, diagnóstico clínico, número de feridas e regiões de ocorrência da UP mais prevalentes.

\section{Captação das imagens fotográficas}

As imagens foram obtidas por câmera fotográfica profissional da marca Canon ${ }^{\circledast}$ modelo T3i - 18-55 mm linha EOS Rebel ${ }^{\circledR}$, com resolução de 18 megapixels e imagem no formato JPEG (Joint Photographic Experts Group).

Durante o registro fotográfico das imagens, a UP do paciente foi posicionada com iluminação de luz solar e com luz artificial desligada para obtenção de melhor qualidade de coloração. Posicionou-se a câmera com eixo perpendicular ao leito da ferida, para evitar distorções resultantes da inclinação da câmera, e com distância de 30 cm da ferida, utilizando-se de uma régua para controle da distância.

\section{Inserção de padrão de área}

Como referência métrica para o cálculo computadorizado da área pelo software ImageJ, foi colocado ao lado da UP um objeto quadrado em papel com $3 \mathrm{~cm}$ de lado. Uma régua foi utilizada para mensurar a distância entre a borda da UP e a lente da câmera fotográfica.

\section{Importação das imagens fotográficas}

As imagens das UP foram importadas pelo software ImageJ, versão 1.47v ano 2012, instalado em um notebook, marca DELL Inspiron 3421. Um único operador realizou quatro aferições em cada uma das imagens: uma sob o modo manual e outras três sob o automático (Brigthness/Contrast, Threshold color e Binary).

\section{Demarcação por meio da técnica manual das imagens fotográficas}

A demarcação sob a técnica manual foi iniciada a partir da abertura do arquivo da imagem. Após a marcação das medidas de interesse, Area/Perimeter, na opção Set Measurements, fez-se a padronização ou calibração da escala, indicando o comprimento conhecido a partir dos eixos $\mathrm{x}$ e y (lados) do objeto-padrão. Foram utilizadas as ferramentas Magnifying glass, Scrolling tool e de seleção Straight. Em seguida, foram preenchidos, na opção Analyse/Set Scale, os espaços referentes à distância e proporção em pixels, e a distância conhecida, ou seja, $3 \mathrm{~cm}$.

A ferramenta de seleção Freehand foi utilizada para o contorno manual da UP, com o auxílio do mouse, sendo considerado como borda o limite do tecido róseo de cicatrização ou da pele íntegra. Após salvar a seleção da borda delineada e ao clicar nas teclas de atalho CTRL+M ou em Analyse/Measure, o software calculou a área $\mathrm{em} \mathrm{cm}^{2}$.

\section{Demarcação por meio da técnica automática das imagens fotográficas}

Foi realizada a análise em técnica automática, utilizando os mesmos procedimentos referentes à transferência das imagens para o computador e a calibração da escala de dimensão horizontal e vertical (x e y).

O cálculo da área pela delimitação da borda da UP pela técnica automática Brightness/Contrast foi realizado adotando-se a sequência de ícones: seleção do objeto de interesse; Edit/Clear Outside, para isolar ao máximo a ferida do restante da imagem; Image/Adjust/Brightness/Contrast; Threshold Color, indicando a opção B\&W; Measure particles, obtendo-se a área final da ferida.

Foi utilizada a opção 8-bits (gray scale), ou escala cinza, no menu Image/Type, que corresponde a um sistema de 256 níveis de cor cinza por pixel. É possível, a partir disto, que a imagem seja convertida em uma representação com números diferentes de zero em apenas dois tons: preto e branco.

Para separação do objeto de interesse utilizou-se a limiarização (Threshold) por meio da técnica Binary ${ }^{(12)}$.

\section{Análise dos dados}

A análise descritiva dos dados, a concordância entre as medidas pelo procedimento de Bland e Altman foram realizadas utilizando o software SPSS, versão 20.0 para Windows, com nivel de significância de $5 \%$.

\section{RESULTADOS}

Avaliou-se 35 UP presentes em 18 pacientes com média e desvio-padrão (DP) de idade igual a 37,1 e 13,9 anos e o tempo médio e DP de lesão de 2,16 e 1,46 meses. Noventa e quatro por cento dos pacientes são do gênero masculino e - Traumatismo Craniano Encefálico foi o diagnóstico clínico prevalente $(66,6 \%)$.

Os 18 participantes apresentaram prevalência média e DP de 2,1 e 1,3 UP por paciente, sendo a maioria localizada nas regiões sacral (25), trocantérica (8) e isquiática (2).

A medida de área da UP realizada pela técnica manual apresentou média e DP de 30,62 e 20,97 cm²; a mensurada pela técnica automática, ferramenta Brightness/Contrast, apresentou média e DP de 27,4 e 20,18 cm²; no modo automático, a ferramenta Threshold apresentou média e DP de 47,6 e $29,9 \mathrm{~cm}^{2}$; no modo automático, a ferramenta Binary apresentou média e DP de 44,34 e $28,38 \mathrm{~cm}^{2}$.

O gráfico de Bland e Altman, com limites superior e inferior de concordância baseados na fórmula (média \pm 2 vezes o DP), mostrou limite superior de $12,24 \mathrm{~cm}^{2}$ e inferior de $-5,78 \mathrm{~cm}^{2}$ ao comparar as medidas da área aferida pelas técnicas manual e automática (Brightness/Contrast), permitindo uma diferença de $18,02 \mathrm{~cm}^{2}$ e representando uma melhor concordância 
entre ambos, quando comparados às demais técnicas, haja vista que os valores estão mais próximos de zero (Gráfico 1).

Gráfico 1: Análise de concordância entre as medidas de área das feridas pelas técnicas manual e automática (Brightness/ Contrast), a partir do gráfico de Bland e Altman $(n=35)$.

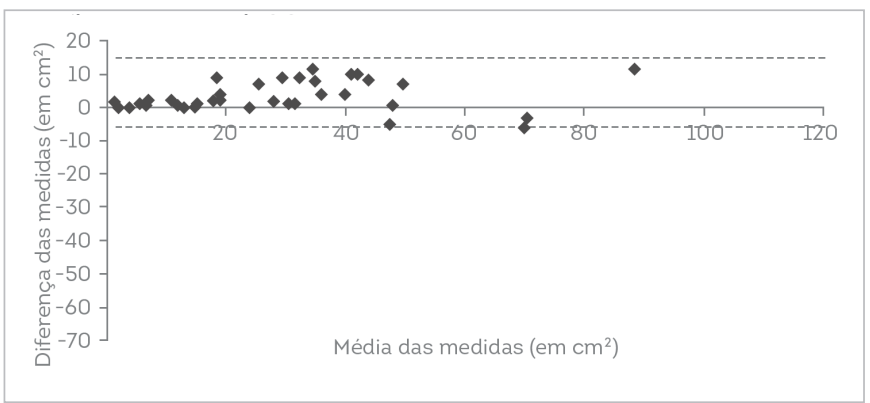

Comparando-se as medidas da área aferida pelas técnicas manual e automática (Threshold) da UP, o limite superior foi de $15,42 \mathrm{~cm}^{2}$ e o inferior de $-49,48 \mathrm{~cm}^{2}$ com uma diferença significativa de $64,9 \mathrm{~cm}^{2}$, representando pouca concordância entre as duas técnicas (Gráfico 2).

Gráfico 2: Análise de concordância entre as medidas de área das UP pelas técnicas manual e automática (Threshold), a partir do gráfico de Bland e Altman ( $n=35)$. Brasilia, DF, Brasil, 2014.

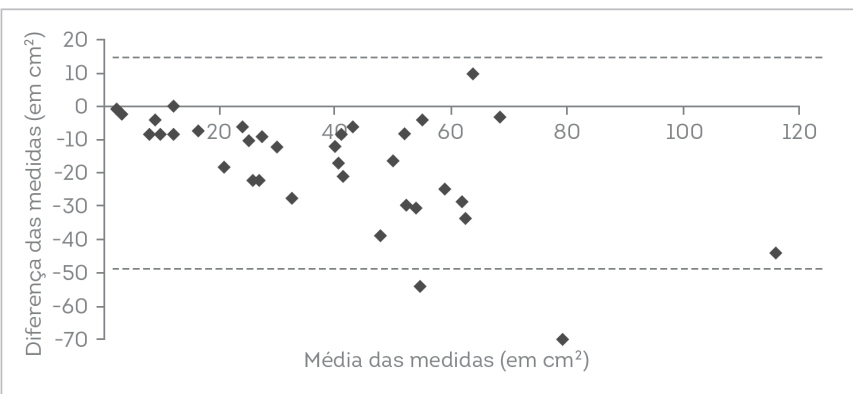

Na comparação entre as medidas da área aferida da UP pelas técnicas manual e automática (Binary), o limite superior foi de $14,17 \mathrm{~cm}^{2}$ e o inferior de $-41,59 \mathrm{~cm}^{2}$, com uma diferença significativa de $55,7 \mathrm{~cm}^{2}$, demonstrando pouca concordância entre as duas técnicas (Gráfico 3).

Gráfico 3: Análise de concordância entre as medidas de área das UP pela técnica manual e automática (Binary), a partir do gráfico de Bland e Altman (n=35). Brasília, DF, Brasil, 2014.

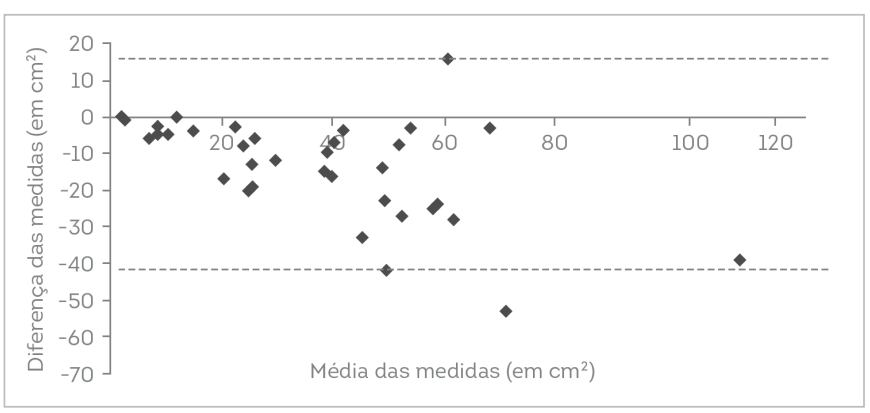

Observou-se a relação proporcional inversa entre a distância dos pontos e o grau de diferença entre as medidas. Aqueles mais próximos de zero representaram uma redução das diferenças e, com isso, uma melhor concordância. Percebeu-se também a influência do tamanho das feridas sobre a localização do ponto, sendo possivel identificá-lo em posição mais distante dos demais, provavelmente atribuída à dificuldade do software em delimitar áreas maiores.

\section{DISCUSSÃO}

Pelo software ImageJ, considerou-se como referência o modo manual de mensuração de área em feridas, sendo demonstrado pelos resultados deste estudo uma melhor concordância com a técnica automática, utilizando a ferramenta Brightness/Contrast. Este achado também foi observado em um estudo que determinou a eficiência e eficácia do referido método computacional automático quando comparado à planimetria digital ${ }^{(13)}$.

As maiores divergências entre as ferramentas Threshold e Binary também foram observadas na comparação de valores entre as médias das medidas, sendo $47,6 \mathrm{~cm}^{2}$ e $44,34 \mathrm{~cm}^{2}$, respectivamente. Esses resultados podem estar relacionados à limitação do software em contornar adequadamente as bordas da ferida e, por isso, adicionar ao cálculo da área os pixels de regiões adjacentes, incluindo os de tecido integro. Por outro lado, houve um melhor resultado referente à ferramenta Brightness/Contrast, com média de $27,4 \mathrm{~cm}^{2}$, ou seja, um valor mais próximo da delimitação manual, com média de $30,62 \mathrm{~cm}^{2}$. A similaridade de cores da ferida às de regiões próximas ao seu contorno, incluindo o esbranquiçamento das áreas internas, foi também observada em um estudo(13), em que se mostrou que não houve diferença estatística entre as mensurações encontradas pela planimetria digital e pela técnica automática ImageJ, ferramenta Brigthness/ Contrast.

Diferentemente de um estudo sobre a utilização de método automatizado, com a ferramenta Threshold(14), não houve equilibrio de contrastes suficiente para determinar a área de interesse com base na distribuição de pixels da imagem.

A ferramenta Binary, também utilizada como recurso adicional para a escala cinza ou gray scale (8-bit) (14), impossibilitou, neste estudo, a ação do operador para graduar os filtros e as cores após o clique sobre o ícone correspondente - Binary/Make Binary.

A variabilidade interobservador durante o contorno manual das UP constitui elemento desfavorável à precisão do método ${ }^{(7,10)}$. Esse problema pode ser minimizado a partir da realização dos procedimentos de análise computacional de todas as imagens por apenas um operador. Ademais, optou-se pela utilização de ferramentas de seleção que 
demonstrassem afinidade pelo contorno e formato real da úlcera, como a forma geométrica elíptica.

Pela técnica automática, a codificação de intensidade em cores e ajustes de contrastes para determinação de área de interesse mostrou-se subjetiva neste estudo, observada a influência da variedade de tons e semitons semelhantes sobre a habilidade e memória visual do operador para a identificação da ferida pós-segmentação.

Considerando aspectos operacionais do software, o presente estudo sugere, além da técnica computacional manual para a mensuração de área de UP, a utilização da ferramenta automática Brightness/Contrast.

Face ao exposto, tornam-se necessários estudos futuros para subsidiar cálculos mais específicos relacionados à área, volume e quantidade de texturas presentes nas feridas, para implementar práticas rotineiras automatizadas, ágeis, precisas e seguras, a serem utilizáveis por qualquer operador.

Uma das limitações do estudo decorre do uso do software ImageJ para contornar com precisão toda a borda da ferida, pois esse procedimento requer controle e habilidade do examinador para realizar a demarcação precisa da UP.
Percebeu-se também que o software apresenta dificuldade para aferição de áreas maiores das UP.

\section{CONCLUSÃo}

Permite-se concluir neste estudo que ambas as técnicas computacionais, manual e automática do software ImageJ. para cálculo de área de UP, demonstraram boa correlação positiva, mas também pouca concordância entre as técnicas, quando comparadas. A ferramenta Brightness/Contrast mostrou medidas aproximadas às da técnica manual. Sugerese que a técnica automática é uma alternativa adicional para a aferição de área de feridas.

Como impacto para a prática clínica na área de saúde e principalmente na Enfermagem, este estudo possibilita avanços computacionais nos procedimentos de demarcação de borda de UP para avaliação de sua área por meio do software ImageJ.

Quanto ao avanço tecnológico, a pesquisa é relevante, pois apresenta resultados importantes, utilizando-se da técnica automática por meio da ferramenta Brightness/ Contrast para mensuração da área de UP.

\section{REFERÊNCIAS}

1. Silva EWNL, Araújo RA, Oliveira EC, Falcão VTFL. Aplicabilidade do protocolo de prevenção de úlcera de pressão em unidade de terapia intensiva. Rev Bras Ter Intensiva 2010;22(2):175-85.

2. Pereira IL, Sousa LC, Santana LA, Guadagnin RV, Neves RS. A posição da câmera fotográfica influencia no cálculo computadorizado da área de úlcera por pressão? Enferm Foc. 2011:2(4):218-21.

3. Lopes AG, Soares MC, Santana LA, Guadagnin RV, Neves RS. Aferição não-invasiva de úlcera por pressão simulada em modelo plano. Rev Bras Enferm 2009:62(2):200-3.

4. Matos LS, Duarte NLV, Minetto RC. Incidência e prevalência de úlcera por pressão no CTI de um Hospital Público do DF. Rev Eletr Enf 2010;12(4):71926.

5. Silva AJ, Pereira SM, Rodrigues A, Rocha AP, Varela J, Gomes LM, Messias N, Carvalhal R, Luís R, Mendes LFP. Custo econômico do tratamento das úlceras por pressão: uma abordagem teórica. Rev Esc Enferm 2013:47(4):971-6

6. Santos CT, Oliveira MC, Pereira AGS, Suzuki LM, Lucena AF. Indicador de qualidade assistencial úlcera por pressão: análise de prontuário e de notificação de incidente. Rev. Gaúcha Enferm 2013:34(1):111-8.

7. Chang AC, Dearman B, Greenwood JE. A Comparison of Wound Area Measurement Techniques: Visitrak@ Versus Photography. Eplasty 2011:11(18):158-66.
8. Reis CLD, Cavalcante JM, Rocha Júnior EF, Neves RS, Santana LA Guadagnin RV, et al. Mensuração de área de úlceras por pressão por meio dos softwares Motic e do AutoCAD®. Rev Bras Enferm 2012;65(2):304-8.

9. Wu GA, MS, Bogie K. Assessment of Gluteus Maximus Muscle Area With Different Image Analysis Programs. Arch Phys Med Rehabil 2009:90:104854.

10. Gardner SE, Frantz RA, Hillis SL, Blodgett TJ, Femino LM, Lehman SM. Volume Measures Using a Digital Image Analysis System are Reliable in Volume Measures Using a Digital Image Analysis
Diabetic Foot Ulcers. Wounds 2012;24(6):146-51.

11. Wild T, Prinz M, Fortner N, Krois W, Sahora K, Stremitzer, Hoelzenbein T. Digital measurement and analysis of wounds based on colour segmentation. Eur Surg 2008; 40(1):5-10.

12. Vardar E, Vardar R, Yükselen V, Makay O, Erkan N, Bayol U, Ozütemiz O. Image-based assessment of esophageal stricture in experimental corrosive esophagitis in animals: An objective, adjunct diagnostic tool. Turk J Gastroenterol 2009:20(1):3-8.

13. Rodrigues DF, Mendes FF, Dias TA, Lima AR, Silva LAF. O programa ImageJ como ferramenta de análise morfométrica de feridas cutâneas. Encicl Biosf 2013:9(17):1955.

14. Shuai W, Yong-rui B, Yun-Peng D, Xian-Sheng M, Ting-Guo K. Evaluation of gastric ulcer model based on gray-scale image analysis. Afr. J. Microbiol. Res 2011:5(11):1285-90. 